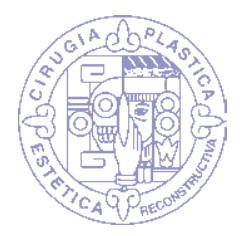

\title{
Injerto cutáneo de unidad estética contralateral para la reconstrucción del párpado superior
}

\author{
Skin graft of contralateral aesthetic unit for reconstruction of the upper eyelid \\ Dr. Heriberto Garza-de la Llave,* Dr. José de Jesús Orozco-Grados, \\ Dr. Carlos Eduardo Rodríguez-Rodríguez, ${ }^{\S}$ Dr. Enrique Chávez-Serna"
}

Palabras clave: Reconstrucción palpebral, defecto palpebral, cirugía funcional, ptosis palpebral, sistema de cierre asistido por vacío, injerto cutáneo.

Keywords: Eyelid reconstruction, eyelid defect, functional surgery, eyelid ptosis, VAC system, skin graft.

\footnotetext{
* Cirujano Plástico y Reconstructivo. División de Cirugía Plástica y Reconstructiva. Hospital Ángeles Metropolitano.

México, Ciudad de México.

‡ Cirujano Plástico y Reconstructivo. División de Cirugía Plástica. Hospital General «Dr. Manuel Gea González», Secretaría de Salud,

México, Ciudad de México.

$\S$ Residente de cirugía plástica y reconstructiva, División de Cirugía Plástica y Reconstructiva, Hospital General «Dr. Manuel Gea González», Secretaría de Salud, México, Ciudad de México.

" Médico pasante en Servicio Social en Investigación. División de Cirugía Plástica, Hospital General «Dr.
}

\section{RESUMEN}

Los párpados representan un elemento complejo cuya función es actuar como barrera física e inmunológica. Su complejidad reside en el parpadeo y en el rango de movimiento que llevan a cabo respetando la integridad de la córnea. El objetivo de este trabajo es proporcionar una alternativa quirúrgica con mínima morbilidad para la reconstrucción del párpado superior, así como dar a conocer las herramientas disponibles para el tratamiento de esta compleja área de la anatomía facial. Presentamos el caso de una mujer de 62 años, con necrosis del $50 \%$ de la superficie del párpado superior y datos de infección. Se inició manejo con desbridamiento quirúrgico, antibioticoterapia sistémica y colocación de sistema con presión negativa. Una vez resuelto el proceso infeccioso y de acuerdo con los principios de reconstrucción por subunidades estéticas, se reconstruyó el $100 \%$ de la subunidad afectada con un injerto cutáneo de espesor total del párpado contralateral obtenido por blefaroplastia, con buenos resultados estético-funcionales. La reparación de los párpados debe ser realizada por un cirujano plástico de manera óptima, utilizando tejidos compatibles, con respecto a composición, tamaño, color y flexibilidad de las estructuras lesionadas, con la finalidad de poder recuperar no sólo la apariencia sino el máximo porcentaje posible de la función.

\section{ABSTRACT}

The eyelids represent a complex element whose function is to act as a physical and immunological barrier. Their complexity lies in the blinking and the range of motion they carry out while respecting the integrity of the cornea. The objective of this work is to provide a surgical alternative with minimal morbidity for the reconstruction of the upper eyelid, as well as to present the tools available for the treatment of this complex area of the facial anatomy. We present the case of a 62-year-old woman, with $50 \%$ necrosis of the upper eyelid surface and evidence of infection. Management was started with surgical debridement, systemic antibiotic therapy, and placement of a negative pressure system. Once the infectious process had been resolved and in accordance with the principles of reconstruction by aesthetic subunits, $100 \%$ of the affected subunit was reconstructed with a full thickness skin graft of the contralateral eyelid obtained by blepharoplasty, with good aesthetic and functional results. The repair of the eyelids must be carried out by a plastic surgeon using compatible tissues, regarding composition, size, color and flexibility of the injured structures, in order to be able to regain not only the appearance, but the maximum percentage possible of the function.

\section{INTRODUCCIÓN}

T os párpados son elementos complejos que ـrindan protección a los ojos de trauma, luz excesiva y mantienen la integridad de la película lagrimal. ${ }^{1}$ Los párpados, a su vez, representan una barrera física e inmunológica en contra de infecciones. ${ }^{2}$ Cualquier pérdida de tejido en esta estructura es particularmente problemática porque el párpado debe mantener su rango de movilidad, flexibilidad, función, así como una superficie de mucosa íntegra para

Citar como: Garza-de la Llave H, Orozco-Grados JJ, Rodríguez-Rodríguez CE, Chávez-Serna E. Injerto cutáneo de unidad estética contralateral para la reconstrucción del párpado superior. Cir Plast. 2020; 30 (3): 167-171. https://dx.doi.org/10.35366/98342 
Manuel Gea González», Secretaría de Salud. México, Ciudad de México.

Recibido: 19 marzo 2020 Aceptado: 12 octubre 2020 estar en contacto con la córnea, que es una estructura sumamente delicada. El parpadeo dinámico y el rango de movimiento a menudo representan un desafío para lograr una reparación adecuada cuando hay pérdida de tejido. Es necesario tener precaución, ya que la incapacidad para cerrar los párpados puede causar pérdida del grado de humedad adecuada del ojo, lo que produce visión borrosa, sensibilidad a la luz y ulceración corneal, así como un mayor riesgo de infección que eventualmente puede llevar a la pérdida del ojo. ${ }^{3}$ Además, el párpado superior representa una de los principales características de la cara, por lo que debe tener una apariencia estética aceptable comparada con el párpado superior contralateral, tomando en cuenta la simetría en la altura del párpado,

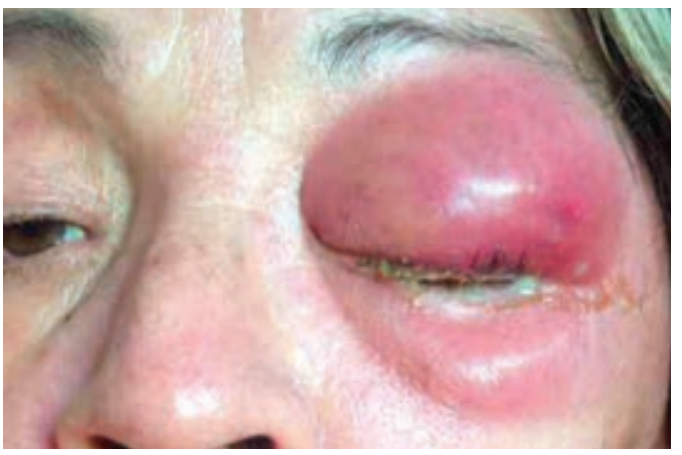

Figura 1: Presentación clínica inicial.
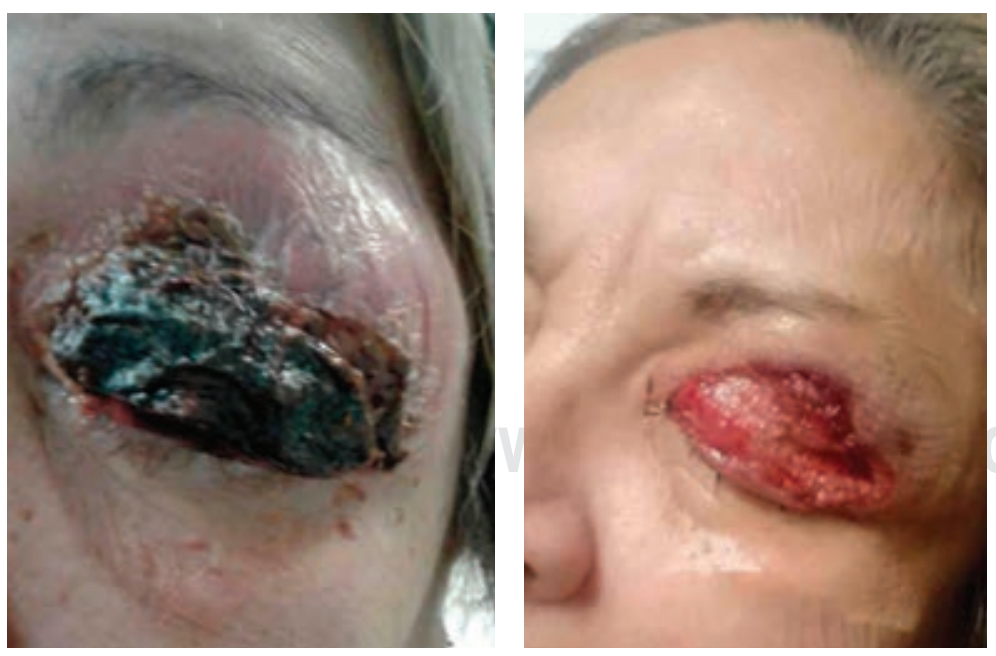

Figura 2: Izquierda: necrosis del párpado superior izquierdo. Derecha: se aprecia pérdida del 50\% del tejido cutáneo después del desbridamiento quirúrgico. la excursión muscular, así como el contorno y los pliegues cutáneos. ${ }^{4}$ La blefaroplastia es un procedimiento quirúrgico en el cual la piel del párpado, el músculo orbicular del párpado y la grasa orbitaria se remodelan con fines principalmente estéticos. ${ }^{5}$ De manera ideal, los defectos de cubierta cutánea de cualquier parte del cuerpo deben reconstruirse con los tejidos coincidentes con respecto a la composición, tamaño, color y flexibilidad, dejando una morbilidad mínima en el sitio del donante y cicatrices discretas. ${ }^{6}$ En cuanto a la reconstrucción palpebral, esto se puede lograr con distintos tipos de colgajos locales o loco-regionales; sin embargo, no siempre se obtienen resultados óptimos. ${ }^{7}$ El injerto de piel de espesor total del párpado representa una alternativa adecuada, especialmente si un colgajo no es factible. En tales casos, el injerto de piel se realiza con frecuencia de sitios donantes clásicos, como la región preauricular, retroauricular o supraclavicular. ${ }^{8}$ En ocasiones, la piel donadora de estos lugares no cumple con las características óptimas, por lo que los resultados no son los ideales. ${ }^{9}$ Las unidades estéticas consisten en áreas limitadas por pliegues y limites naturales; éstas se subdividen en subunidades basándose en la complejidad anatómica de algunas zonas, como los párpados o la nariz, por lo que es posible cerrar defectos con un injerto cutáneo de espesor total tomado de la unidad estética contralateral correspondiente. ${ }^{10} \mathrm{Al}$ dividir la cara en segmentos cutáneos de diferente color, textura y grosor, cantidad de grasa subcutánea, movilidad y distribución de pelo, facilita la reconstrucción de estos segmentos, tomando injertos lo más parecidos al área afectada. ${ }^{11}$ Están descritas nueve unidades estéticas faciales: frente, nariz, párpado, mejilla, labio superior, labio inferior, mentón, oreja, piel cabelluda, que a su vez, se dividen en subunidades. Existen recomendaciones generales para obtener mejores resultados al reparar estas unidades; sin embargo, no existe una guía estandarizada para determinar cuál es la mejor opción para la reparación de cada unidad estética.

\section{CASO CLÍNICO}

Mujer de 62 años que inició su padecimiento de forma súbita, con edema importante, eri- 
Figura 3:

\section{Colocación de sistema con presión negativa vacuum assisted closure con presión intermitente a 125 mmHg.}

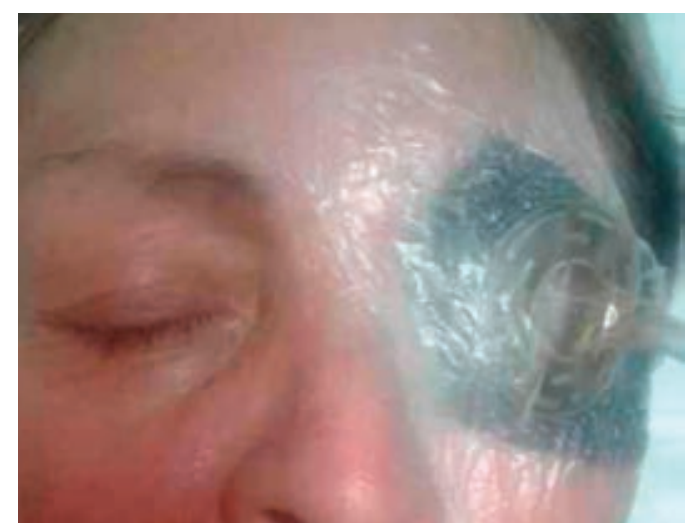

tema, secreción purulenta y dolor en párpado superior izquierdo, de ocho horas de evolución, sin antecedente de trauma o infección (Figura 1). Presentaba imposibilidad para el movimiento del párpado, específicamente limitación para la apertura, lo que condicionaba la visión con ese ojo. Decidió manejarlo con fomentos de agua tibia sin observar mejoría, por lo que acudió al Servicio de Urgencias una semana después de haber iniciado con la sintomatología. Durante la valoración en urgencias, se observó presencia de necrosis cutánea del párpado comprometido mayor al 50\% (Figura 2), de acuerdo a la valoración del Servicio de Oftalmología, sin compromiso del globo ocular, por lo que se inició tratamiento antibiótico con tobramicina solución oftálmica; se realizó aseo quirúrgico con desbridamiento, corroborando la integridad del músculo elevador del párpado, así como del músculo orbicular del párpado y se decidió colocar sistema de terapia con presión negativa vacuum assisted closure (VAC) intermitente a $125 \mathrm{mmHg}$, utilizando la esponja negra de poliuretano (VAC GranuFoam), con recambios cada tres días, completando una terapia total de tres recambios (Figura 3). Al retirar el VAC se observó zona de pérdida cutánea en el párpado superior mayor al $50 \%$ de la superficie, bien delimitada, con adecuado tejido de granulación e integridad del músculo elevador del párpado y del orbicular, sin datos de infección activa y resultados de cultivos negativos (Figura 4).

Tomando en cuenta que la paciente ya no presentaba infección y que la lesión se había delimitado, comprometiendo únicamente la lámina anterior del párpado, se decidió realizar procedimiento quirúrgico para reconstruir subunidades afectadas mediante injerto cutáneo de espesor total.

En la evaluación del caso, se planteó la posibilidad de que en un solo procedimiento, utilizando anestesia local, se realizara blefaroplastia derecha y utilizar el tejido resecado como injerto de espesor total para reconstruir la unidad contralateral. Al presentar compromiso de más del $50 \%$ del párpado, y tomando en cuenta los principios reconstructivos de acuerdo con las subunidades estéticas, fue necesario reconstruir el $100 \%$ de la subunidad afectada mediante un injerto cutáneo de espesor total del párpado contralateral (Figura 5).

La paciente se presentó a consulta subsecuente con buena evolución, adecuada función de ambos párpados y buen resultado estético en el postquirúrgico a seis meses (Figura 6).

\section{DISCUSIÓN}

Cuando se presenta una lesión del párpado superior que representa más del $30 \%$ de la subunidad estética, no se recomienda su cierre primario, por lo que se debe recurrir a alguna otra técnica para reconstruir esta subunidad estética.

La reconstrucción del párpado superior con un injerto cutáneo de espesor total representa una buena alternativa con respecto a otros mé-

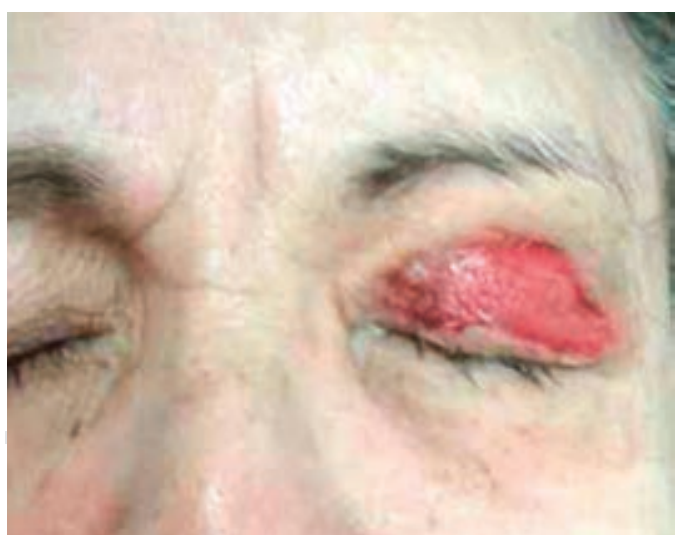

Figura 4: Retiro de sistema con presión negativa, con integridad del músculo del elevador del párpado y del orbicular. Se observa tejido de granulación sin datos de infección. 

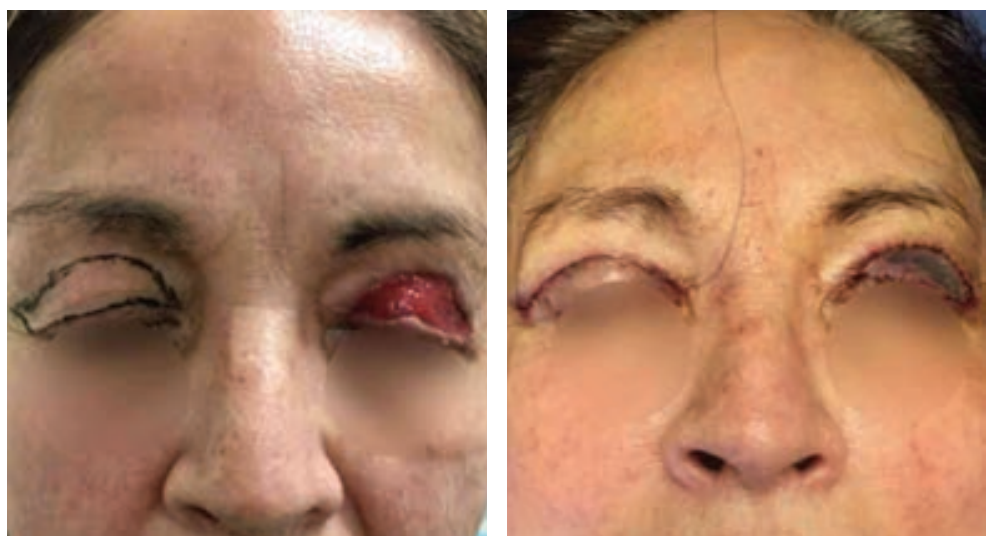

Figura 5: Izquierda: planeación prequirúrgica. Derecha: postquirúrgico inmediato.
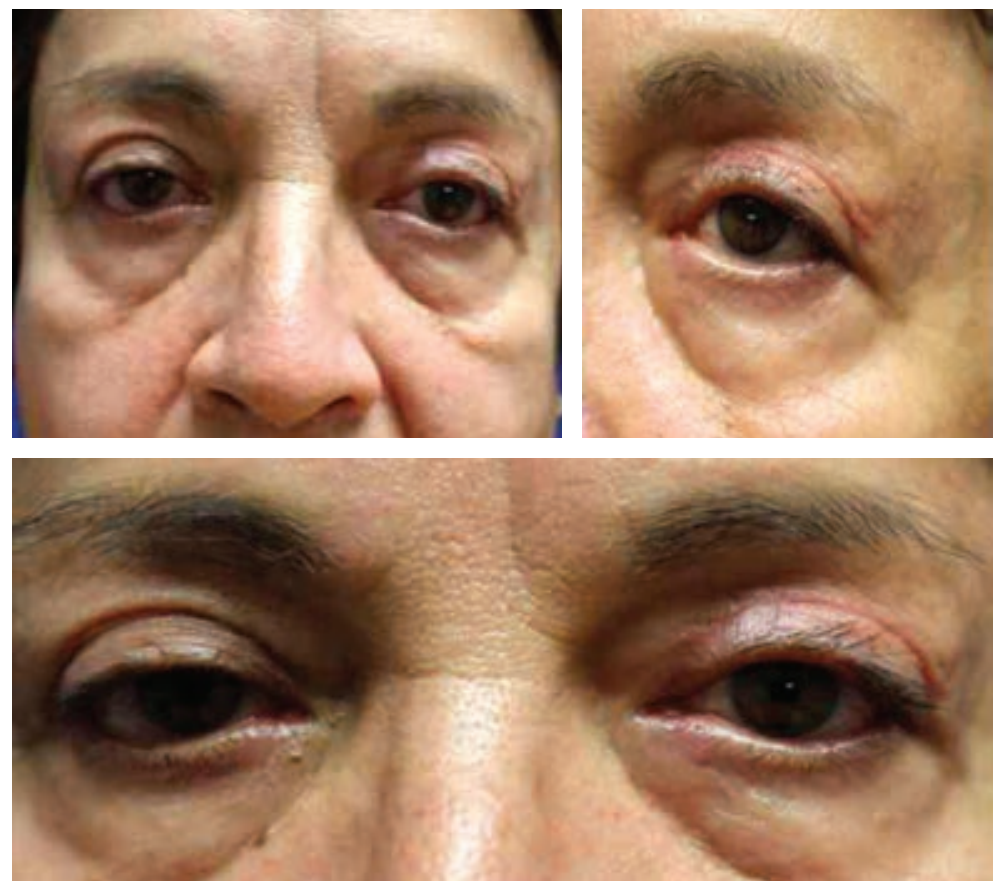

Figura 6: Resultado postquirúrgico a los seis meses.

todos de reparación, en casos en los que sólo se ha afectado la lámina anterior y no existe compromiso muscular.

Es difícil lograr buenos resultados al reconstruir el párpado superior. A pesar de que existen varias técnicas descritas, el uso de colgajos cutáneos laterales de rotación eleva el riesgo de dañar fibras del nervio facial que pueden ocasionar parálisis del músculo orbicular, causando a su vez lagoftalmos.
También está descrito el intercambio de párpados que moviliza las capas de tejido de forma compuesta, desde el párpado inferior ipsilateral al defecto. Ejemplos de este principio son la técnica de Cutler-Beard y la de Mustardé, ambos requieren un procedimiento secundario para dividir el colgajo y sacrificar tejido del párpado inferior.

Otra opción incluye técnicas sin puente cutáneo que dependen de un colgajo lamelar anterior sostenido por un injerto lamelar posterior o injertos bilamelares, con índice de complicaciones altos que incluyen: lagoftalmos, queratopatías, retracción palpebral y entropión marginal.

También hay opciones con colgajos tomados de la frente; sin embargo, las cicatrices son muy evidentes y comprometen el resultado final.

Se presenta un caso con una lesión que tiene como consecuencia un defecto a reparar, mismo que requiere un procedimiento para reconstruir el parpado, en el cual se toma ventaja de las circunstancias de la unidad estética contralateral y se toma un injerto cutáneo de espesor total contralateral sin comprometer la función; de esta manera, este procedimiento con fines meramente reconstructivos se convierte en un procedimiento estético.

Al tomar el injerto del párpado sano, se corrige un problema estético de blefarocalasia, mientras se obtiene una opción reconstructiva para el lado afectado. De la misma forma se utiliza un mismo procedimiento con fines estéticos, como la blefaroplastia, para solucionar la blefarocalasia y a su vez reconstructivo en cuanto a tomar la redundancia cutánea de la blefarocalasia para resolver la pérdida de la subunidad estética del párpado.

El párpado contralateral representa una buena opción, ya que la piel del área donadora carece de pelo, tiene un grosor ideal, una textura y color semejantes al nativo y la cicatriz de la unidad estética donadora es prácticamente imperceptible.

\section{CONCLUSIÓN}

La técnica presentada constituye una alternativa viable, ya que es rápida, con poca morbilidad y muy buenos resultados, además ofrece un 
beneficio estético y reconstructivo, por lo que se le debería prestar más atención, ya que no existen muchos reportes al respecto.

\section{REFERENCIAS}

1. Subramanian N. Reconstructions of eyelid defects. Indian J Plast Surg 2011; 44 (1): 5.

2. Lin L, Martin J. State of the art in congenital eyelid deformity management. Facial Plast Surg 2016; 32 (2): 142-149.

3. Espinoza GM, Prost AM. Upper eyelid reconstruction. Facial Plast Surg Clin N Am 2016; 24 (2): 173-182.

4. Morley AMS, deSousa J-L, Selva D, Malhotra R. Techniques of upper eyelid reconstruction. Surv Ophthalmol 2010; 55 (3): 256-271.

5. Bhattacharjee K, Misra D, Deori N. Updates on upper eyelid blepharoplasty. Indian J Ophthalmol 2017; 65 (7): 551.

6. Mathijssen IMJ, van der Meulen JC. Guidelines for reconstruction of the eyelids and canthal regions. J Plast Reconstr Aesthet Surg 2010; 63 (9): 1420-1433.

7. Takahashi Y, Mito H, Nakamura Y, Kakizaki H. Upper eyelid reconstruction by making a skin defect similar to skin removal in blepharoplasty. J Craniofac Surg 2014; 25 (5): e445-446.

8. Tuncali D, Ates L, Aslan G. Upper eyelid full-thickness skin graft in facial reconstruction. Dermatol Surg 2005; 31 (1): 65-70

9. Meissner M, Hofmann W, Kaufmann R. Reconstruction of the upper eyelid: full-thickness skin grafting between the same contralateral aesthetic units. J Dtsch Dermatol Ges 2016; 14 (3): 319-320.

10. Russo F, Linares M, Iglesias ME, Martínez-Amo JL, Cabo F, Tercedor J et al. Técnicas reconstructivas de elección por unidades estéticas faciales. Actas DermoSifiliográficas 2017; 108 (8): 729-737.

11. González-Ulloa M. Regional aesthetic units of the face. Plast Reconstr Surg 1987; 79 (3): 489-490.

Correspondencia:

Dr. Heriberto Garza de la Llave

Calzada de Tlalpan Núm. 4800,

Col. Belisario Domínguez Sección 16, 14080

Alcaldía Tlalpan, México, Ciudad de México.

E-mail: dr.garzadelallave@gmail.com

Conflicto de intereses: Los autores declaran no tener conflicto de intereses. 